\section{RADIOMIC MARKERS ASSOCIATED WITH CLINICAL BENEFIT IN ADVANCED UVEAL MELANOMA PATIENTS WITH RADIOGRAPHIC PROGRESSION ON TEBENTAFUSP}

'Volkan Beylergil, '2Laura Collins, 'Lawrence Schwartz, 'Thomas Eche, 'Binsheng Zhao, ${ }^{1}$ Richard Carvajal, ${ }^{2}$ Shaad Abdullah, ${ }^{1}$ Volkan Beylergil ${ }^{*}$, 'Laurent Dercle. 'Columbia University Medical Center, New York, NY, United States; ${ }^{2}$ Immunocore, Abingdon, United Kingdom

Background Tebentafusp, a bispecific fusion protein consisting of affinity-enhanced $\mathrm{T}$ cell receptor targeting a gp100 derived peptide fused to anti-CD3 effector, has shown overall survival (OS) benefit in untreated metastatic uveal melanoma (mUM). The OS benefit derives from all RECIST response categories, even progressive disease (PD). In $\mathrm{Ph} 2$ trial of previously treated mUM (NCT02570308), one-third (35\%) of 48 evaluable patients with best response of PD had ctDNA reduction ( ${ }^{3} 0.5$ log reduction) and longer OS (median 16.9 months) compared to the group without ctDNA reduction (median OS 8.5 months).

Methods 34 of 127 mUM patients from Ph2 trial ${ }^{1}$ were selected based on best response of $\mathrm{PD}$ and no ctDNA reduction (Group A, $\mathrm{n}=17$ ) or $0.5 \log$ ctDNA reduction (Group B, $n=17)$. One patient per group were excluded due to poor image quality or limited CT/MRI sequences. Tumor lesions were manually segmented on CT and MRI. Radiomics features were extracted at baseline and Week-8 (first assessment). The objective was to use unsupervised machine-learning to develop two signatures using 16 features to classify the two groups. The per-patient analysis signature $(n=32)$ combined 8 volumetric features on CT-scan at baseline and change by Week-8. The per-lesion analysis signature $(n=148)$ combined 4 features (volume and 3 radiomics features previously associated with outcome to checkpoint immunotherapy in cutaneous melanoma) at two timepoints using CT and MRI. Performance was evaluated using area under the receiver operating characteristic curve (AUC).

Results The median OS for Groups A and B were 8.5 and 16.9 months, respectively. In the per-patient analysis, a volumetric signature classified patients into the groups with AUC 0.71 (95\%CI: $0.53-0.90$ ) with $63 \%$ specificity and $81 \%$ sensitivity at the optimal threshold (0.57). In the per-lesion analysis, a radiomic signature reached an AUC of 0.70 (95\%CI: $0.58-0.81$ ) with $66 \%$ specificity and $74 \%$ sensitivity at the optimal threshold (0.53). Group B had lower baseline tumor lesion volume $(\mathrm{AUC}=0.65)$, distinct baseline tumor heterogeneity $(\mathrm{AUC}=0.66)$, and distinct change in tumor heterogeneity by week 8 (AUC $=0.66 / 0.69$ on CT/MRI

Conclusions A radiomic analyses of a subset of PD patients was able to predict Group B, patients with ctDNA reduction and longer OS, at a patient and lesion level. The strongest radiomic predictor by $\mathrm{CT} / \mathrm{MRI}$ was decrease on treatment in tumor heterogeneity. Confirmation in a larger dataset of these signatures is needed to identify which patients may be benefiting from tebentafusp despite radiographic progression.

Trial Registration NCT02570308

\section{REFERENCE}

1. Sacco JJ, Carvajal R, Butler MO, et al. A phase (ph) II, multi-center study of the safety and efficacy of tebentafusp (tebe) (IMCgp100) in patients (pts) with metastatic uveal melanoma (mUM). Ann Oncol 2020;31:S1442-S1143.

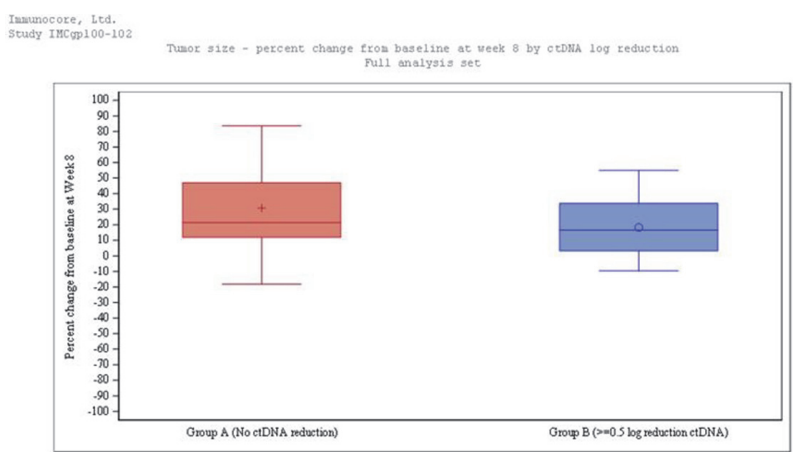

Abstract 819 Figure 1 Percent change in tumor measurement from baseline at week 8 per independent review committee by Group A and

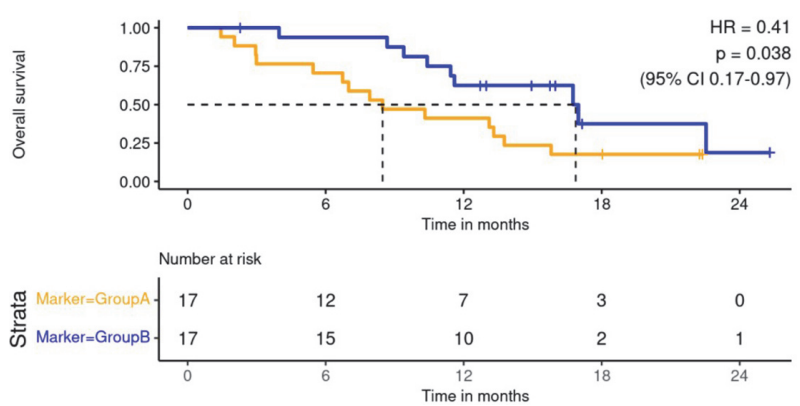

Abstract 819 Figure 2 Kaplan-Meier plot comparing overall survival rates in group $A$ and group $B$ patients

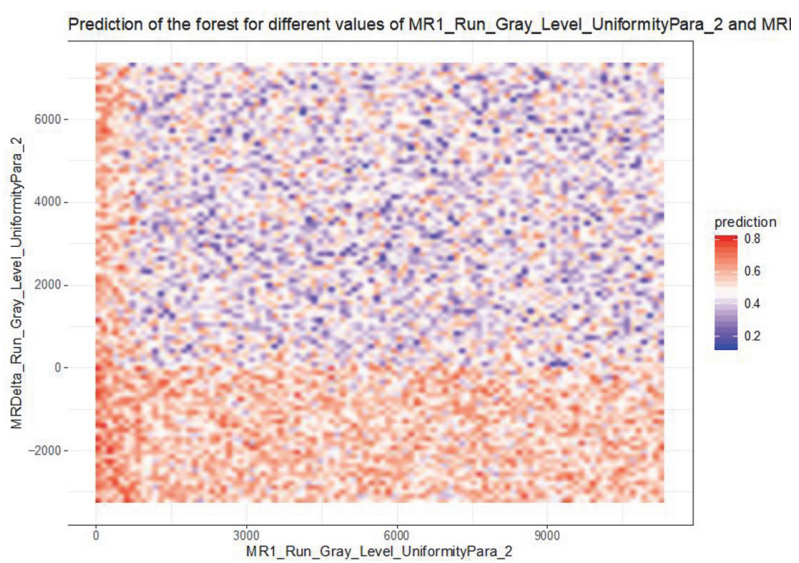

Abstract 819 Figure 3 Blue color represents a high probability of the patient being in Group A while red color indicates high probability of being in Group B

http://dx.doi.org/10.1136/jitc-2021-SITC2021.819 